\title{
NÚMERO DE CASOS DE HANSENÍASE NO BRASIL DE 2010 A 2015
}

\section{ARTIGO ORIGINAL}

MONTEIRO, Lis Mayra Dantas¹, FACCO, Lucas², FECURY, Amanda Alves³ ARAÚJO, Maria Helena Mendonça de ${ }^{4}$, OLIVEIRA, Euzébio de ${ }^{5}$, DENDASCK, Carla Viana $^{6}$, SOUZA, Keulle Oliveira da7 ${ }^{7}$ DIAS, Claudio Alberto Gellis de Mattos ${ }^{8}$

MONTEIRO, Lis Mayra Dantas. Et al. Número de casos de hanseníase no Brasil de 2010 a 2015. Revista Científica Multidisciplinar Núcleo do Conhecimento. Ano 05, Ed. 11, Vol. 25, pp. 62-70. Novembro de 2020. ISSN: 2448-0959, Link de acesso: https://www.nucleodoconhecimento.com.br/saude/hanseniase-no-brasil, $\quad$ DOI: 10.32749/nucleodoconhecimento.com.br/saude/hanseniase-no-brasil

\section{RESUMO}

A Hanseníase é considerada uma doença infectocontagiosa, causada pela bactéria Mycobacterium leprae conhecida também como Bacilo de Hansen. Além de ser uma doença com evolução gradativa, a hanseníase depende da relação entre o parasita e o hospedeiro. O objetivo deste trabalho foi mostrar o número de casos de Hanseníase no Brasil entre 2010 a 2015 no Brasil. A pesquisa foi realizada no DATASUS (http://datasus.saude.gov.br/). Com o avanço das políticas públicas o número de casos da doença do Brasil reduziu entre os anos de 2010 a 2015.

\footnotetext{
${ }^{1}$ Técnica em Mineração, egressa do Instituto Federal do Amapá (IFAP).

2 Discente do Curso de Medicina da Universidade Federal do Amapá (UNIFAP).

${ }^{3}$ Biomédica, Doutora em Doenças Tropicais, Professora e pesquisadora do Curso de Medicina da Universidade Federal do Amapá (UNIFAP).

${ }^{4}$ Médica, Professora e pesquisadora do Curso de Medicina da Universidade Federal do Amapá (UNIFAP).

${ }^{5}$ Biólogo, Doutor em Doenças Topicais, Professor e pesquisador do Curso de Educação Física da Universidade Federal do Pará (UFPA).

${ }^{6}$ Teóloga, Doutora em Psicanálise, pesquisadora do Centro de Pesquisa e Estudos Avançados- CEPA.

${ }^{7}$ Socióloga, Mestranda em Estudos Antrópicos na Amazônia, Integrante do Grupo de Pesquisa "Laboratório de Educação, Meio Ambiente e Saúde" (LEMAS/UFPA).

${ }^{8}$ Biólogo, Doutor em Teoria e Pesquisa do Comportamento, Professor e pesquisador do Programa de PósGraduação em Educação Profissional e Tecnológica (PROFEPT), Instituto Federal do Amapá (IFAP).
}

$\mathrm{RC}: 66577$

Disponível em: https://www.nucleodoconhecimento.com.br/saude/hanseniase-no- 
Apresenta maior prevalência no sexo masculino e, a maior taxa de doentes encontra-se em maiores faixas etárias, ou seja, em adultos. O período de incubação da doença (que pode variar de 2 a 7 anos). Em vista disso, vários problemas podem ser deflagrados a nível individual, como acometimentos físicos debilitantes e psicológicos. Tendo em vista o intuito de diminuir o número de casos da doença no país e conseguir fazer o diagnóstico o mais precoce possível, devem ser analisados os achados clínicos e os exames disponíveis para essa detecção.

Palavras-Chave: Epidemiologia, hanseníase, Mycobacterium leprae.

\section{INTRODUÇÃO}

A Hanseníase é considerada uma doença infectocontagiosa, pois precisa do contato direto com o bacilo para que seja transmitida entre pessoas. É causada pela bactéria Mycobacterium leprae conhecida também como Bacilo de Hansen. Essa enfermidade só é detectada, em média, entre 2 a 7 anos de incubação e os sintomas costumam aparecer de 11 a 16 dias. Nesta fase sintomática é possível a transmissão interpessoal, e normalmente, apenas uma pequena parcela adoece (BRASIL, 2002; OLIVEIRA et al., 2016).

A Hanseníase pode ser classificada em duas formas: A hanseníase Paucibacilar ou a hanseníase Multibacilar. Na variação Paucibacilar, o doente tem resistência aos bacilos, apresentando até cinco lesões na pele. Os bacilos se encontram em menor número no organismo, não sendo suficiente para transmissão a outras pessoas. Neste caso existe a possibilidade de cura por maneira espontânea. A hanseníase Multibacilar compõe o chamado grupo transmissor, com seis ou mais lesões cutâneas. Os enfermos não apresentam defesa contra a bactéria, eliminando-as pelas vias aéreas (BRASIL, 2002; BRASIL, 2016). O contágio normalmente ocorre pelo contato direto entre uma pessoa suscetível e um doente não tratado, normalmente algum familiar (BRASIL, 2015).

$\mathrm{RC}: 66577$

Disponível em: https://www.nucleodoconhecimento.com.br/saude/hanseniase-nobrasil 
Além de ser uma doença com evolução gradativa, a hanseníase depende da relação entre o parasita e o hospedeiro. Quando o hospedeiro tem um sistema de defesa eficiente, ele tende a não manifestar a doença. Por vários motivos como vulnerabilidade socioeconômica e o convívio com muitas pessoas em um mesmo ambiente, ela pode atingir indivíduos de várias idades e ambos os sexos (BRASIL, 2002).

Os sintomas podem aparecer na pele ou no sistema nervoso. Na pele costumam ser caracterizados por lesões com perda ou aumento de sensibilidade. Podem aparecer manchas pigmentares, placas, infiltrações, tubérculos e/ou nódulos. No sistema nervoso os sintomas podem aparecer como incapacidades físicas e, dependendo do grau da doença, deformidades nas extremidades do corpo (BRASIL, 2002; FREITAS, 2015).

O tratamento da hanseníase é feito com a combinação de várias drogas, denominada poliquimioterapia ou PQT. Para os pacientes com a forma Paucibacilar costuma-se utilizar dois tipos de antibióticos. Para os que apresentam forma Multibacilar recomenda-se três tipos de antibióticos (BRASIL, 2002; FREITAS, 2015).

Segundo o Ministério da Saúde, a quantidade registrada de casos novos no Brasil durante os anos pesquisados foi de 193.021 enfermos. (BRASIL, 2017).

\section{OBJETIVO}

Mostrar o número de casos de Hanseníase no Brasil entre 2010 a 2015 no Brasil.

\section{MÉTODO}

A pesquisa foi realizada no DATASUS (http://datasus.saude.gov.br/), sendo retirados dados de Hanseníase a partir das seguintes etapas: na aba "Acesso à Informação",

RC: 66577

Disponível em: https://www.nucleodoconhecimento.com.br/saude/hanseniase-no- 
selecionou-se "Informações de Saúde (TABNET)" e logo após clicou-se em "Epidemiológicas e Morbidade". Na próxima página ao clicar em "Casos de Hanseníase - Desde 2001 (SINAN)", escolheu-se a opção "Hanseníase - desde 2001", e em abrangência geográfica optou-se por "Brasil por Região e Unidade da Federação". Foram retirados dados de 2010 a 2015, escolhendo: em linha as opções "Ano diagnóstico", "Região", "Sexo", "Faixa Etária", "Modo detecção", "Avaliação diagnóstica" e "Lesão Cutânea". No campo coluna a opção "Não ativa" e em conteúdo a frase "Pacientes por Atendimento" foram utilizadas para as opções de linha acima. Após a seleção, esses dados foram compilados na ferramenta Excel, componente Office da Microsoft Corporation. Pesquisa bibliográfica realizada no laboratório do Instituto Federal de Educação, Ciência e Tecnologia do Amapá, Campus Macapá, situado na: Rodovia BR 210 KM 3, s/n - Bairro Brasil Novo. CEP: 68.909-398, Macapá, Amapá, Brasil.

\section{RESULTADOS}

A figura 1 mostra o número de casos de hanseníase no Brasil entre 2010 e 2015. Sendo observado que nos últimos anos houve uma diminuição do número de casos, com um pequeno aumento no quantitativo de 2014 em relação ao ano de 2013.

RC: 66577

Disponível em: https://www.nucleodoconhecimento.com.br/saude/hanseniase-no- 
Figura 1 Número de casos de hanseníase no Brasil entre 2010 e 2015.

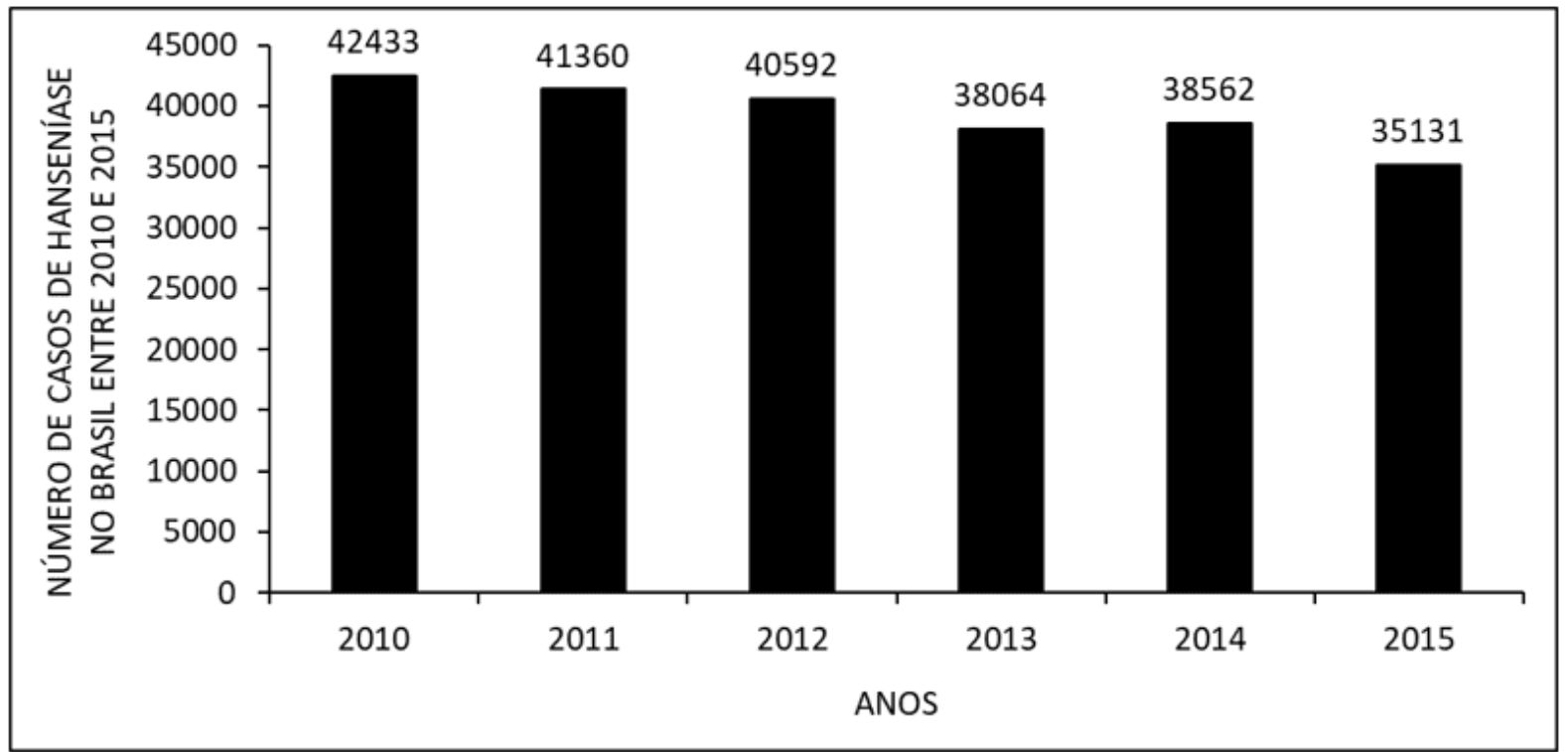

A figura 2 mostra o número de casos de hanseníase no Brasil entre 2010 e 2015, por regiões do país. Os dados mostram que a maior quantidade de atendimentos ocorre no Nordeste e a minoria no Sul.

Figura 2 Mostra o número de casos de hanseníase no Brasil entre 2010 e 2015, por regiões do país.

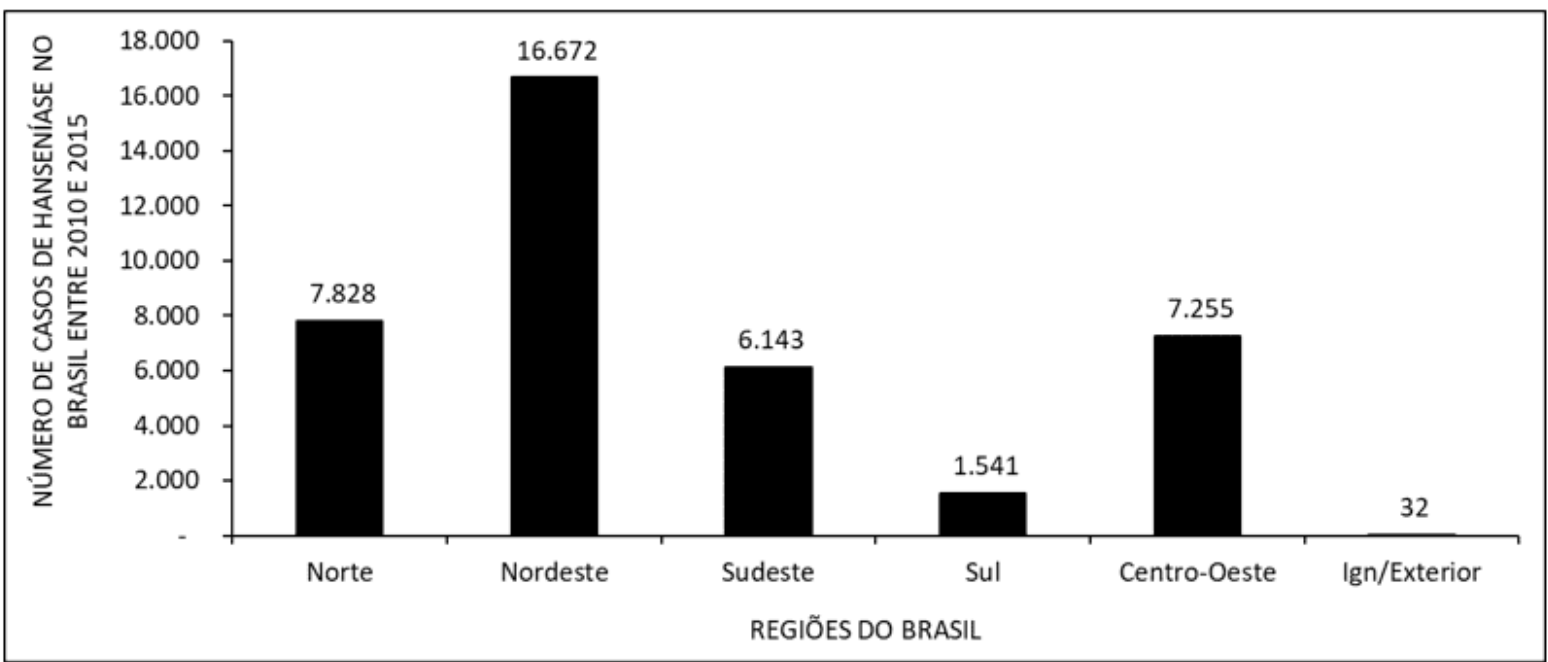

RC: 66577

Disponível em: https://www.nucleodoconhecimento.com.br/saude/hanseniase-no- 
A figura 3 mostra o número de casos de hanseníase no Brasil entre 2010 e 2015, de acordo com o gênero. Onde observa-se que há um quantitativo maior de ocorrências em pessoas do sexo masculino quando comparadas às pessoas do gênero feminino.

Figura 3 Mostra o número de casos de hanseníase no Brasil entre 2010 e 2015, de acordo com o gênero.

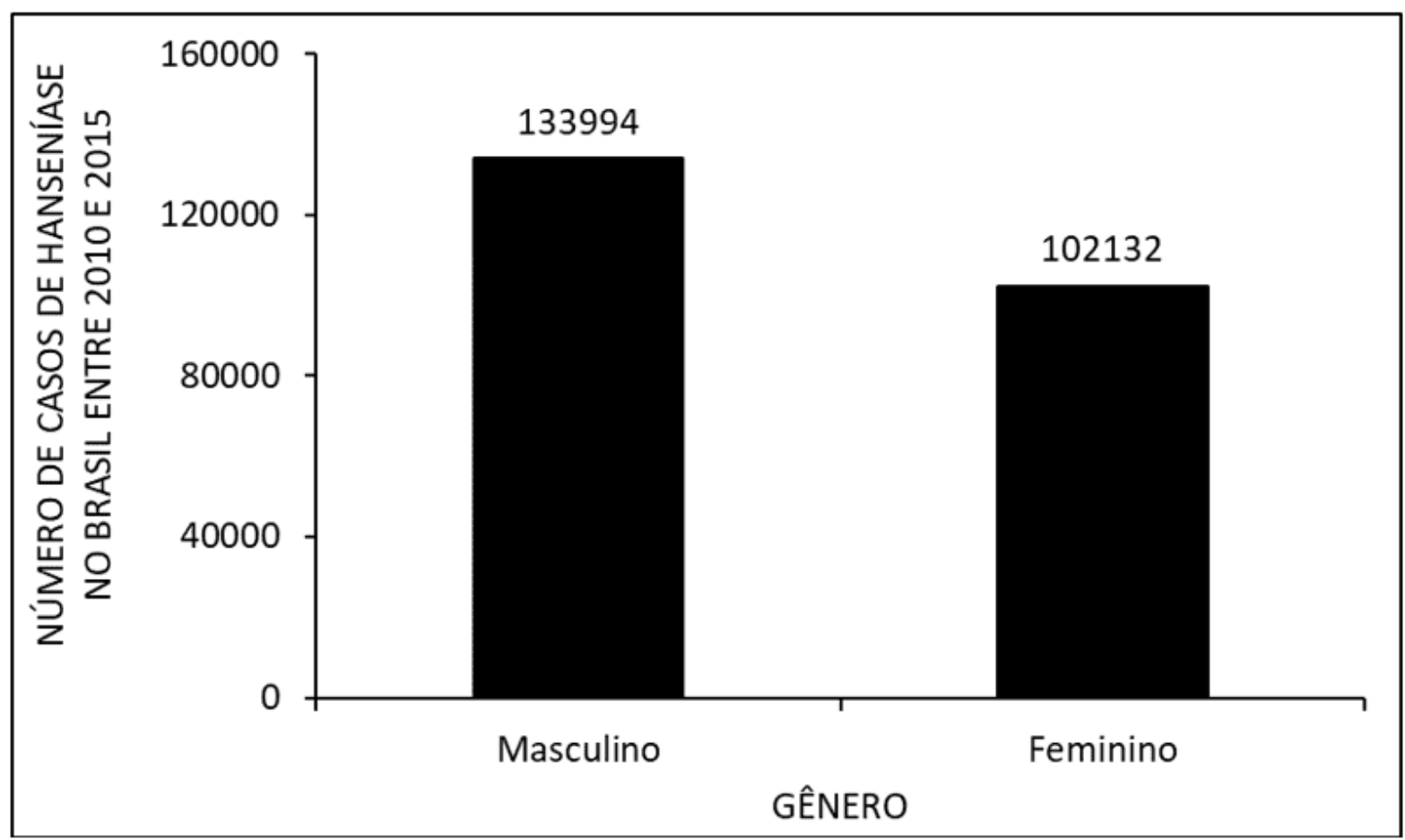

A figura 4 mostra o número de casos de hanseníase no Brasil entre 2010 e 2015, por faixa etária. Onde nota-se uma taxa maior de ocorrência da doença em adultos na idade de 20 a 64 anos, e menor em crianças de 1 a 9 anos e idosos de 80 anos ou mais.

RC: 66577

Disponível em: https://www.nucleodoconhecimento.com.br/saude/hanseniase-no- 
Figura 4 Mostra o número de casos de hanseníase no Brasil entre 2010 e 2015, por faixa etária.

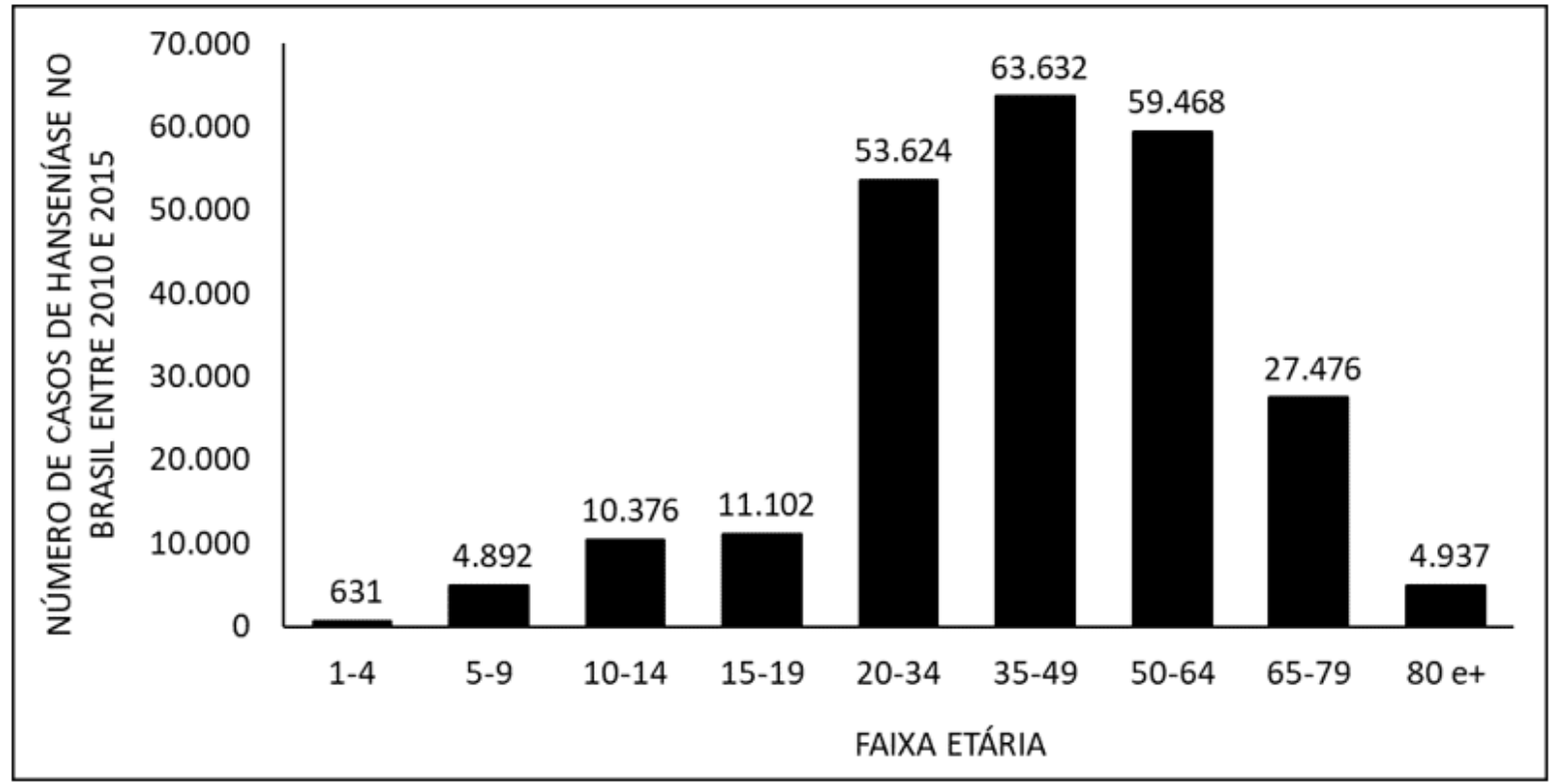

A figura 5 mostra o número de casos de hanseníase no Brasil entre 2010 e 2015, de acordo com o modo de detecção. O principal modo de detecção da doença são os encaminhamentos, seguidos pela demanda espontânea.

RC: 66577

Disponível em: https://www.nucleodoconhecimento.com.br/saude/hanseniase-no- 
Figura 5 Mostra o número de casos de hanseníase no Brasil entre 2010 e 2015, de acordo com o modo de detecção.

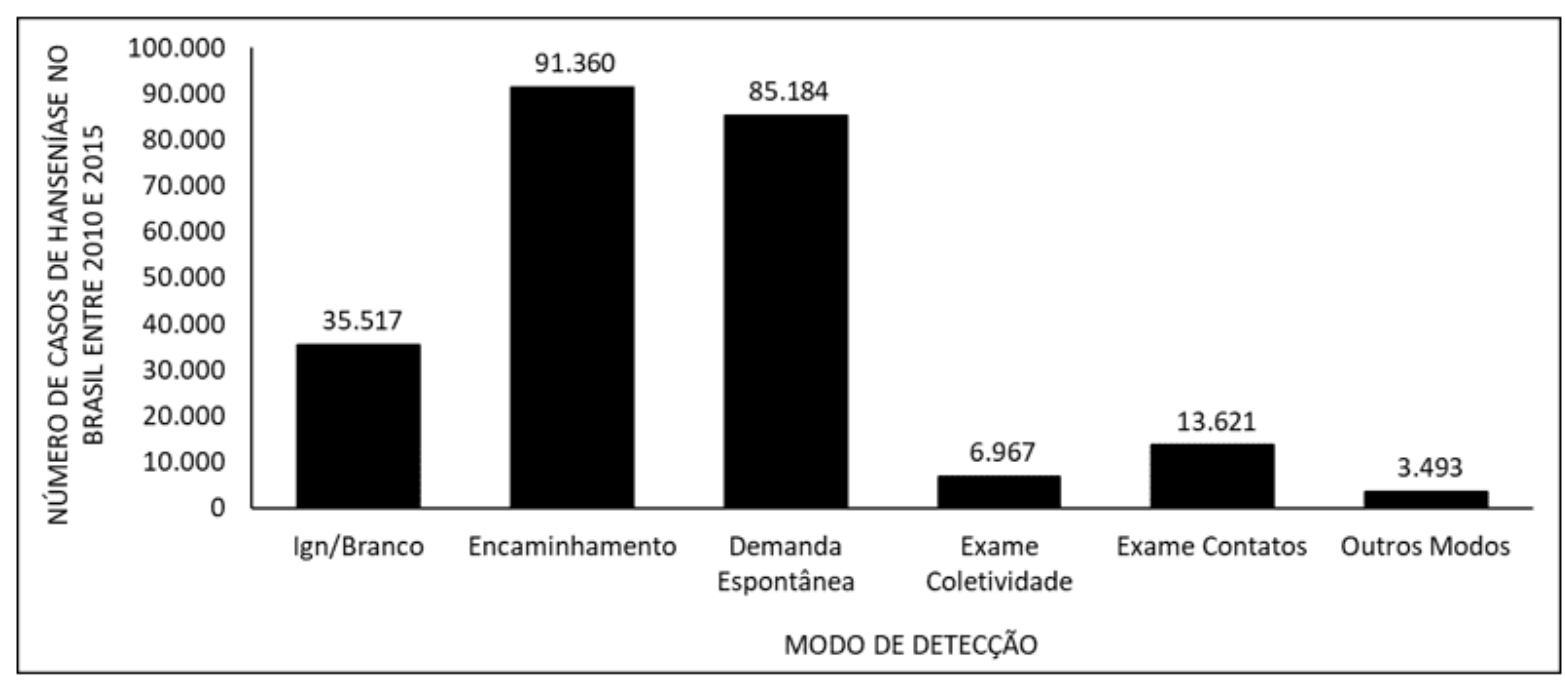

A figura 6 mostra o número de casos de hanseníase no Brasil entre 2010 e 2015, por tipos de lesão cutânea. Os dados mostram a maioria dos pacientes tem de 2 a 5 lesões cutâneas e minoria não apresenta qualquer lesão.

RC: 66577

Disponível em: https://www.nucleodoconhecimento.com.br/saude/hanseniase-no- 
Figura 6 Mostra o número de casos de hanseníase no Brasil entre 2010 e 2015, por tipo de lesão cutânea.

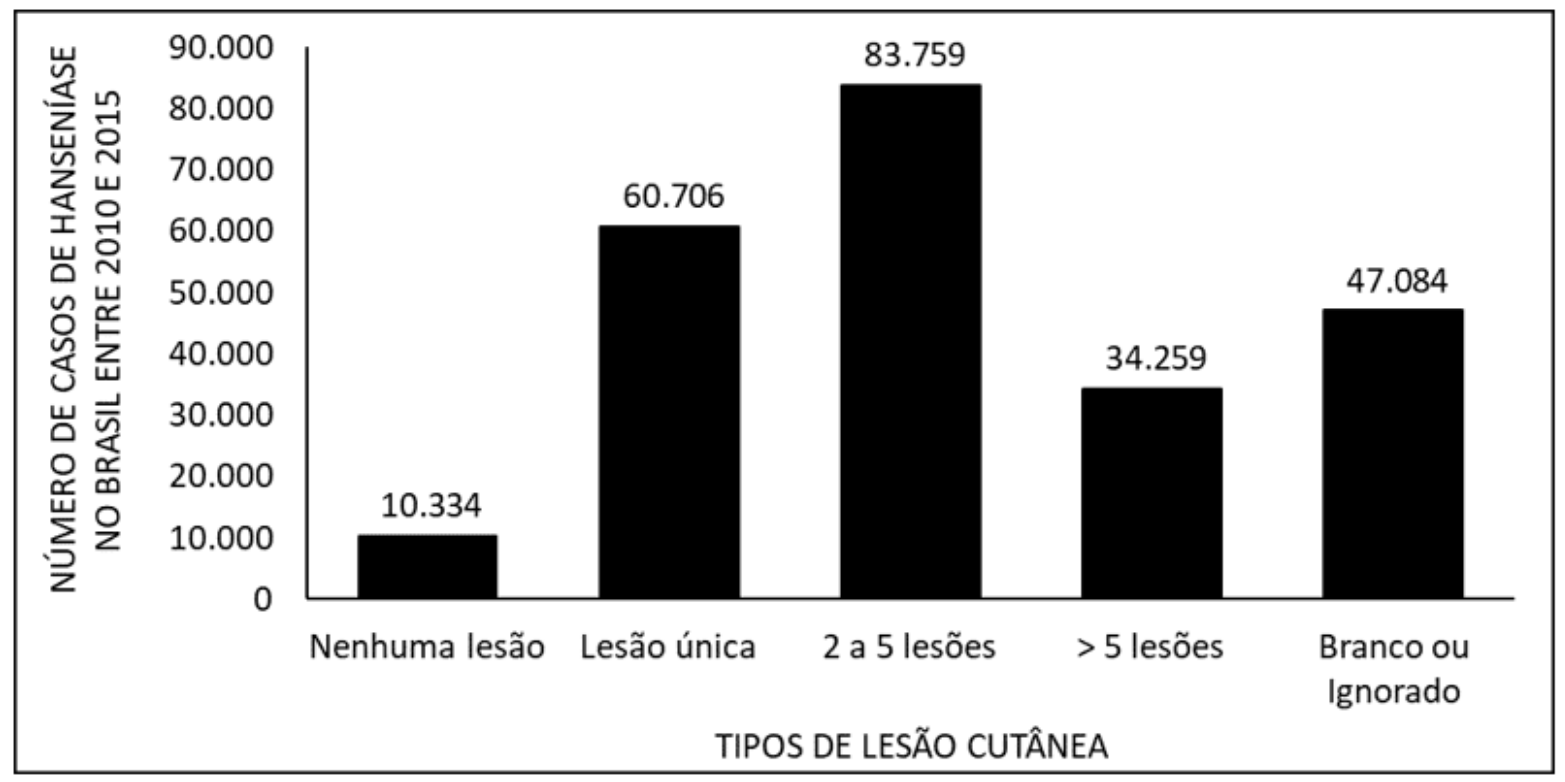

A figura 7 mostra o número de casos de hanseníase no Brasil entre 2010 e 2015, por avaliação diagnóstica. Os dados mostram que a maioria dos casos são pertencentes ao grau zero e a minoria ao grau II da doença.

RC: 66577

Disponível em: https://www.nucleodoconhecimento.com.br/saude/hanseniase-no$\underline{\text { brasil }}$ 
Figura 7 Mostra o número de casos de hanseníase no Brasil entre 2010 e 2015, por avaliação diagnóstica.

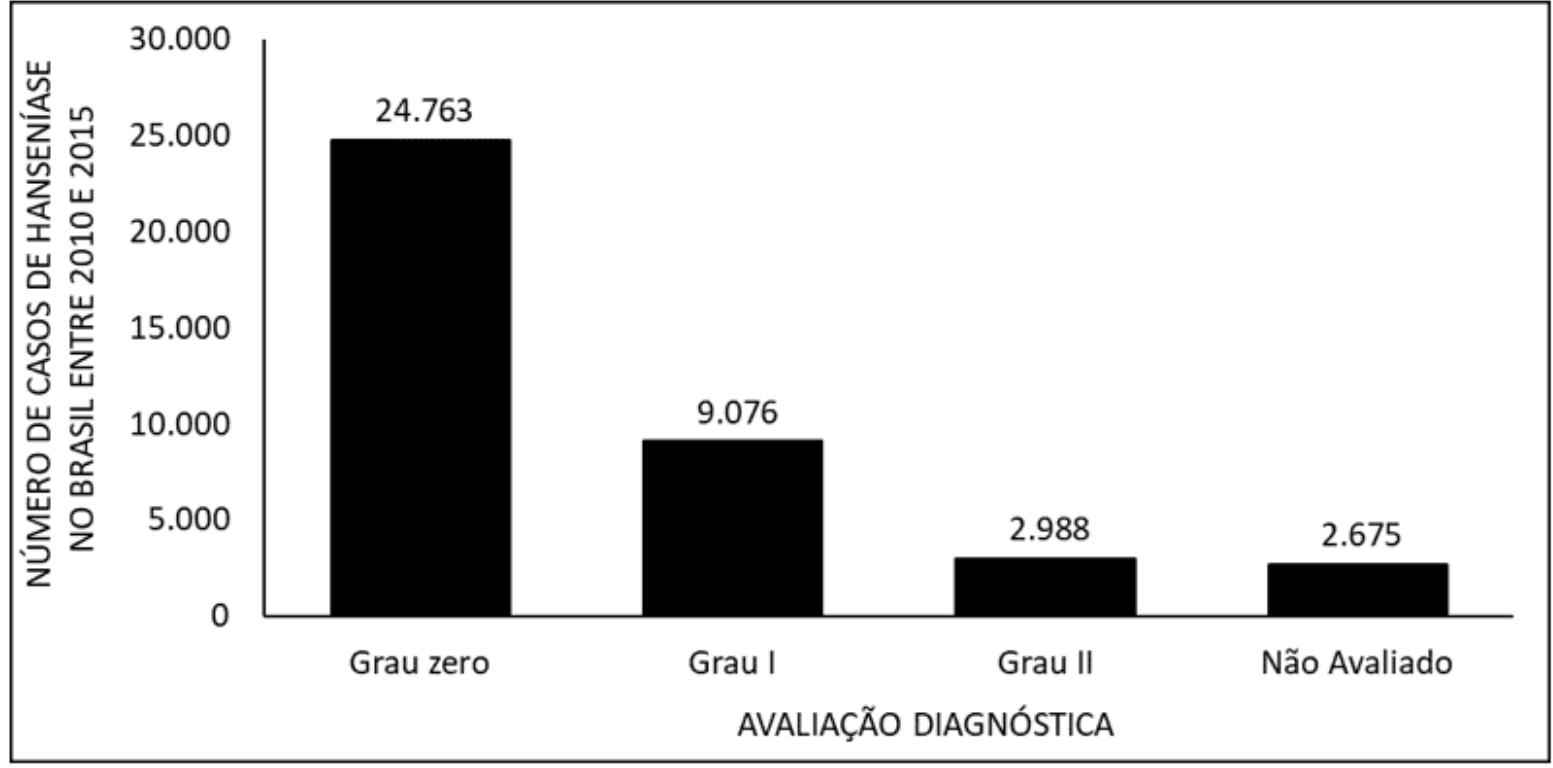

\section{DISCUSSÃO}

Durante os anos de 2010 a 2015 observa-se que houve uma diminuição no número de pacientes atendidos com hanseníase, com um pequeno aumento no ano de 2014 (figura 1). Os dados são corroborados pela literatura. A diminuição provavelmente ocorreu pela implantação de políticas públicas para o enfrentamento da doença, como diagnóstico precoce e tratamento bem-sucedido, o que conduz à cura. $\mathrm{O}$ aumento no quantitativo de 2014 provavelmente deve-se a campanha de detecção de novos casos da doença (BRASIL, 2015; 2017; 2017a).

O número de casos de hanseníase mostra mais atendimentos na região nordeste e menos na região sul (figura 2). Segundo a literatura esse alto número de infectados no Nordeste deve-se provavelmente a alta taxa de transmissão, que ocorre nas épocas festivas. Momento em que as unidades básicas de saúde não atuam. $\mathrm{Na}$ região Sul a provável causa do baixo índice de enfermos, refere-se a distância entre

$\mathrm{RC}: 66577$

Disponível em: https://www.nucleodoconhecimento.com.br/saude/hanseniase-no- 
os serviços de saúde e o paciente, dificultando o acesso e colaborando para a evolução da doença, a um grau de incapacidade física elevado (ROCHA, 2015).

De acordo com o gênero, durante os anos de 2010 a 2015, os homens tiveram maior incidência da doença do que as mulheres (figura 3). Ao comparar os dados com a literatura, observa-se que a provável causa deste alto índice se deve ao fato dos homens se exporem mais a doença do que as mulheres, demorando a procurar os serviços de saúde e abandonando o tratamento antes da cura. Isso o torna suscetível a desenvolver a deformidade característica da doença (ARAGOSO et al., 2014; ARANTES et al., 2010).

A nível de ocorrência, os indivíduos de maior faixa etária predominam entre os contaminados. Um dos fatores prevalentes para esse aspecto é o tempo de incubação da bactéria (Mycobacterium leprae), o qual pode se estender de dois até sete anos e, dessa forma, apresentando-se na vida adulta e, juntamente a isso, acaba por culminar em possíveis acometimentos psicológicos, uma vez que, com o aparecimento de problemáticas físicas - amplificadas pela demora do diagnóstico o paciente pode se ver incapacitado de exercer sua atividade profissional e, por conseguinte, dificuldade em contribuir economicamente em âmbito familiar. Além disso, com o acontecimento de casos familiares, pode-se aumentar a chance de adoecimento de crianças (VELÔSO, 2018).

Para que haja o diagnóstico da hanseníase, e sua consequente classificação e estadiamento, são analisados vários aspectos. Tais fatores verificados podem variar mediante a classificação empregada, sendo duas das principais, a de Ridley e Jopling toma como base as características histopatológicas, clínicas, baciloscópicas e imunológicas; a classificação da Organização Mundial da Saúde (OMS) emprega o índice baciloscópico em sua classificação, estabelecendo que, caso haja no máximo cinco lesões a nível cutâneo e tronco nervoso, tem-se a hanseníase Paucibacilar. No caso de haver mais de cinco lesões, a nível cutâneo e tronco nervoso, tem-se então a hanseníase multibacilar. Porém, caso haja disponibilidade de realizar a

RC: 66577

Disponível em: https://www.nucleodoconhecimento.com.br/saude/hanseniase-no- 
baciloscopia no paciente e contate-se o resultado positivo, a classificação é automaticamente pautada em multibacilar (LASTÓRIA e ABREU, 2012).

A depender da forma de manifestação da hanseníase, vários acometimentos podem estar presentes de forma física no indivíduo contaminado cujo bacilo está em atividade, dentre eles: lesões granulomatosas (as quais podem surgir em diferentes formatos, como nódulos ou pápulas), edemas, sintomas ocasionados quando os troncos nervosos são afetados (como atrofia dos músculos, fraqueza e dor), problemas imunológicos, debilidades sensoriais, motoras, processos inflamatórios agudos e sistêmicos e outras implicações para o organismo (PESSOA, 2019).

A hanseníase, com o intuito de que não venha a deflagrar acometimentos graves para o indivíduo, deve ser diagnosticada precocemente, utilizando a lógica clínica e os exames preconizados. Caso o diagnóstico ocorra de forma tardia, maiores podem ser as implicações. Além disso, a epidemiologia e seu respectivo controle são necessários para que haja conhecimento amplo da situação de prevalência da doença, refletindo, dessa forma, no contingente de casos, pois, caso o controle não seja eficaz, pode haver maior disseminação da doença e, por conseguinte, aumento significativo no número de casos. A procura por auxílio médico no estágio inicial da doença culmina na prevalência de casos pertencentes ao grau zero (no qual os segmentos musculares e suas respectivas sensibilidades encontram-se preservadas, caracterizando, em ampla parte das vezes, um bom prognóstico do paciente) (PESSOA, 2019; BRASIL, 2018).

\section{CONCLUSÃO}

Com o avanço das políticas públicas que visam combater a hanseníase, pautadas na precocidade do diagnóstico e do tratamento, o número de casos da doença do Brasil, a nível majoritário, reduziu entre os anos de 2010 a 2015.

RC: 66577

Disponível em: https://www.nucleodoconhecimento.com.br/saude/hanseniase-no- 
A hanseníase apresenta maior prevalência no sexo masculino, sendo que a provável causa dessa realidade é a maior exposição de homens a essa patologia, uma vez que a procura pelo serviço de saúde se dá de forma menos abrangente e, além disso, do maior abandono do tratamento, acarretando no avanço da doença e o consequente surgimento de debilidades orgânicas do indivíduo.

Além disso, a maior taxa de doentes encontra-se em maiores faixas etárias, ou seja, em adultos, sendo o principal fator responsável por isso o período de incubação da doença (que pode variar de 2 a 7 anos). Em vista disso, vários problemas podem ser deflagrados a nível individual, como acometimentos físicos debilitantes e psicológicos, e familiar, pois, com o surgimento da debilidade, podem surgir dificuldades econômicas em decorrência da perda da atividade profissional remunerada.

Tendo em vista o intuito de diminuir o número de casos da doença no país e conseguir fazer o diagnóstico o mais precoce possível, devem ser analisados os achados clínicos e os exames disponíveis para essa detecção. Caso o paciente consiga ter seu diagnóstico feito no estágio inicial da doença, costuma-se haver bom prognóstico, sendo que sua saúde orgânica pode ser muito preservada, mantendo a sensibilidade e integridade de seus segmentos musculares.

\section{REFERÊNCIAS}

ARAGOSO, I.; CARVALHO, R.M.B.; SOUSA, C.M.; VIEIRA, G.D. Hanseníase em Rondônia: incidência e características dos casos notificados, 2001 a 2012. Epidemiologia e Serviços de Saúde, v.23, n.2, Brasília, jun. 2014.

ARANTES, C.K.; FILIPE, M.S.; GARCIA, M.L.R.; NARDI, M.T.; PASCHOAL, V.D.A. Avaliação dos serviços de saúde em relação ao diagnóstico precoce da hanseníase. Epidemiologia e Serviços de Saúde, v.19, n.2, Brasília, jun. 2010.

RC: 66577

Disponível em: https://www.nucleodoconhecimento.com.br/saude/hanseniase-no$\underline{\text { brasil }}$ 
BRASIL. Ministério da Saúde. Secretaria de Políticas de Saúde. Departamento de Atenção Básica. Guia para o controle da hanseníase. $1^{\underline{a}}$ ed., Brasília (DF): Ministério da Saúde, 2002. Disponível em: <http://bvsms.saude.gov.br/bvs/publicacoes/guia_de_hanseniase.pdf> Acesso em: 24 ago 2017.

BRASIL. Ministério da Saúde. Secretaria de Vigilância em Saúde. Departamento de Vigilância das Doenças Transmissíveis. Diretrizes para vigilância, atenção e eliminação da Hanseníase como problema de saúde pública: manual técnicooperacional. $1^{\underline{a}}$ ed., Brasília (DF): Ministério da Saúde, 2016. Disponível em: $<$ http://www.saude.pr.gov.br/arquivos/File/DiretrizesdoManuaTcnicoOperacionaldeH ansenase.pdf> Acesso em: 05 set 2017.

BRASIL. Ministério da Saúde. Secretaria de Vigilância em Saúde. Departamento de Vigilância das Doenças Transmissíveis. Eliminar a hanseníase é possível: um guia para os municípios. Brasília (DF): Ministério da Saúde, 2015. Disponível em: $<$ http://bvsms.saude.gov.br/bvs/publicacoes/eliminar_hanseniase_possivel_versao_p reliminar.pdf> Acesso em: 05 set 2017.

BRASIL. Boletim Epidemiológico. Brasilía DF. Ministério da Saúde. 49, n. 4, 12p. 2018.

FREITAS, A.A. Resposta imune celular e humoral a proteínas recombinantes do Mycobacterium leprae em pacientes com outras dermatoses. Tese (Doutorado) Universidade Federal de Goiás, Instituto de Patologia Tropical e Saúde Pública, Program de Pós Graduação em Medicina Tropical e Saúde Pública, Goiânia, 2015. Disponível em: $<\mathrm{https}: / /$ posstrictosensu.iptsp.ufg.br/up/59/o/AlinedeAraujoFreitas_2015_vers\%C3\%A3ofinal. pdf> Acesso em: 12 set 2017.

RC: 66577

Disponível em: https://www.nucleodoconhecimento.com.br/saude/hanseniase-nobrasil 
LASTÓRIA, J. C; ABREU, M. A. M. M. Hanseníase: diagnóstico e tratamento. Diagn Tratamento, v. 17, n. 4, p. 173-179, 2012.

OLIVEIRA, Ciane Martins de; et. al. A evolução da assistência ao paciente com Hanseníase: dos leprosários à poliquimioterapia. Revista Científica Multidisciplinar Núcleo do Conhecimento. Ano 01, Vol. 06, Ed. 08, pp. 68-80. Agosto de 2016. ISSN: 2448-0959

PESSOA, M. M. S. F. S. Hanseníase no Brasil: Uma revisão literária, nos anos de 2014 a 2019. 45p. Trabalho de Conclusão de Curso (Graduação de Farmácia) Universidade Federal do Rio Grande do Norte, Rio Grande do Norte, Natal, 2019.

ROCHA, A.C.A.A. Análise da sazonalidade da incidência de hanseníase segundo regiões geográficas, climas e biomas do Brasil. 2015. 82 f. Dissertação (Mestrado em Ciências Ambientais) - Universidade do Estado de Mato Grosso, Programa de Pós-Graduação em Ciências Ambientais, Cáceres/MT.

VELÔSO, D. S. Perfil clínico-epidemiológico da hanseníase no estado do Piauí, no período de 2009 a 2016. 127p. Dissertação (Mestrado em Medicina Tropical) Instituto Oswaldo Cruz, Piauí, Teresina, 2018.

Enviado: Novembro, 2020.

Aprovado: Novembro, 2020.

RC: 66577

Disponível em: https://www.nucleodoconhecimento.com.br/saude/hanseniase-no- 